\title{
Salpingectomy for ectopic pregnancy: Does length really matter?
}

\section{Jonathan Gaughran*, Sian Mitchell, Tom Holland}

Department of Gynaecology, Guys and St Thomas' Hospitals, London, United Kingdom

Received: 14 September 2020

Accepted: 29 October 2020

\section{*Correspondence:}

Dr. Jonathan Gaughran,

E-mail: jonathan.gaughran@gstt.nhs.uk

Copyright: (C) the author(s), publisher and licensee Medip Academy. This is an open-access article distributed under the terms of the Creative Commons Attribution Non-Commercial License, which permits unrestricted non-commercial use, distribution, and reproduction in any medium, provided the original work is properly cited.

\section{ABSTRACT}

This abstract describes a case of a tubal stump ectopic. The aetiology, presentation and management of such rare cases are described. The evidence bases for the rationale for leaving a short versus a long stump at salpingectomy is reviewed.

Keywords: Ectopic, Tubal, Stump, Salpingectomy, Interstitial, Isthmus

\section{INTRODUCTION}

Ectopic pregnancy is defined as a pregnancy that implants outside of the uterine cavity- $90 \%$ of which are located in the fallopian tube. ${ }^{1}$ Tubal stump ectopic is a pregnancy in the remnant fallopian tube after salpingectomy. According to limited data, the incidence ranges from 0.4 to $1.6 \%$ of all ectopic pregnancies. ${ }^{2,3}$ The only recognised risk factor for tubal stump ectopic is assisted reproductive techniques. Mortality from stump ectopic pregnancies is $10-15$ times higher than tubal ectopic pregnancy (2.0-2.5\% compared to $0.14 \%)$ and so prevention and early detection is paramount. ${ }^{4}$ Ectopic pregnancies typically present with a triad of amenorrhea, abdominal pain and vaginal bleeding. ${ }^{5}$ Atypical presentations may mimic gastrointestinal disease. The only feature in the presentation to raise suspicion of stump ectopic is a history of salpingectomy. Given the reduced ability of the tubal remnant to distend, patients may present with hemoperitoneum and/or hypovolaemic shock. ${ }^{5}$ An interstitial pregnancy (IP) occurs when the blastocyst implants in the most proximal section of the fallopian tube (the interstitial portion), which is within the myometrium. ${ }^{6}$ The isthmic portion of the tube is adjacent to the interstitial portion and is also the site of anastomosis of uterine and ovarian vessels and so incision into this region is associated with marked blood loss. ${ }^{2}$ With no evidence that leaving a short or minimal remnant stump at salpingectomy actually decreases the incidence of stump ectopic, it should be considered that leaving a longer stump may reduce the probability of implantation in the isthmic or interstitial portion.

\section{CASE REPORT}

A 31-year-old female self-presented to the early pregnancy unit with a 5-day history of lower abdominal pain and a positive pregnancy test. By last menstrual period she was 6 weeks and 1-day gestation. The pain was described as 'mild', had not localised, and was not associated with fever, gastrointestinal or genitourinary symptoms. Observations were within normal limits and abdominal examination unremarkable. Six months prior to this the patient had undergone an uncomplicated salpingectomy at the same hospital for an ectopic pregnancy. She reported two prior first trimester miscarriages which did not require medical intervention and no live births. There was no significant medical history or other previous surgery. A transvaginal ultrasound scan (TVUS) was performed the same day by a level 2 sonographer. The scan reported an endometrial thickness of $22 \mathrm{~mm}$ with no evidence of intra or extrauterine pregnancy. There was no free fluid and the scan was tolerated well. Human chorionic gonadotropin (hCG) 
was $46 \mathrm{IU} / \mathrm{l}$ and progesterone $31 \mathrm{nmol} / \mathrm{l}$. 48 hours later hCG was $132 \mathrm{IU} / 1$ (2.8xincrease). At this point the patient reported ongoing mild lower abdominal pain that did not require analgesia. She denied vaginal bleeding. In line with the local protocol a plan was made by the oncall consultant to repeat the ultrasound scan 5 days later as long as the patient remained well. Follow up TVUS showed no evidence of an intra or extra uterine pregnancy. The right ovary contained two corpora lutea (Figure 1). A differential diagnosis of ovarian ectopic was considered. There was no free fluid and the scan was tolerated well. Repeat hCG was 2709 IU/l.

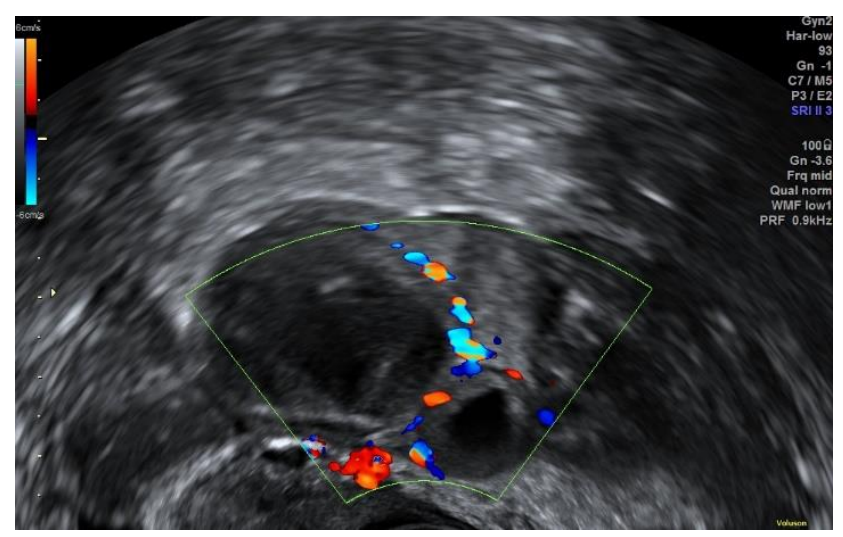

Figure 1: Right ovary containing haemorrhagic corpus lutem and a smaller cystic corpus luteum with circumferential doppler signal or 'ring of fire sign'.

The on-call consultant elected to re-scan the patient 48 hours later. At follow up the patient complained of worsening pain. A TVUS revealed a hyperechoic mass adjacent to the uterus. The mass had an anechoic centre with a second hyperechoic ring in keeping with a gestational sac and yolk sac (Figure 2). As there was no myometrium surrounding the mass, an interstitial ectopic was thought to be unlikely. There was still no free fluid nor marked tenderness. hCG was now 6744 IU/L. The patient was counselled and consented for laparoscopic management of ectopic pregnancy and taken to theatre the same day.

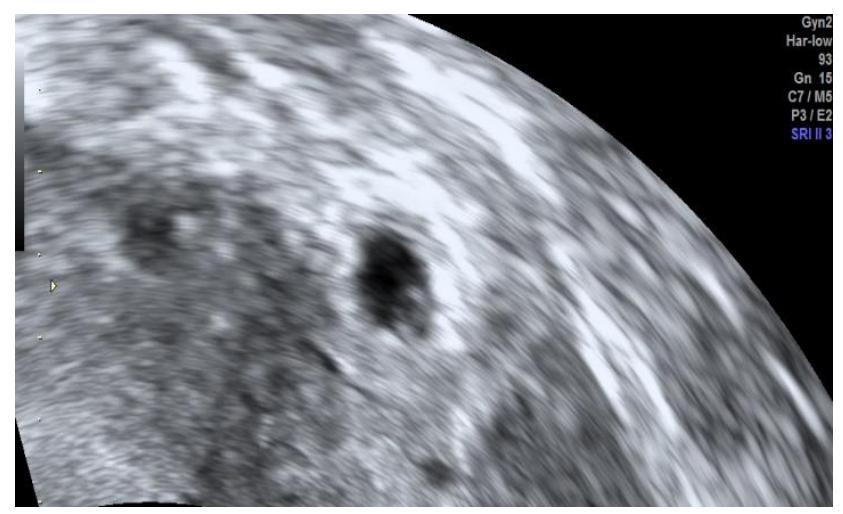

Figure 2: Hyperechoic ring within the cystic mass in keeping with a yolk sac.
At laparoscopy a left sided ectopic was seen in the isthmic portion of the tubal remnant, almost within the interstitial portion (Figure 4). To reduce blood loss 20 units of argipressin diluted in $10 \mathrm{ml}$ of sodium chloride was injected into the stump base. An attempt to pass an endo-loop led to rupture of the ectopic which was subsequently retrieved in an endo-bag. Bipolar diathermy was applied to the base and one figure of eight suture with 2-0 vicryl thrown to secure haemostasis. Blood loss was less than $50 \mathrm{ml}$, the patient was discharged the following day and hCG 5 days later had decreased to 7 IU/L. The patient was counselled about potential recurrence and offered early TVUS in future pregnancies.

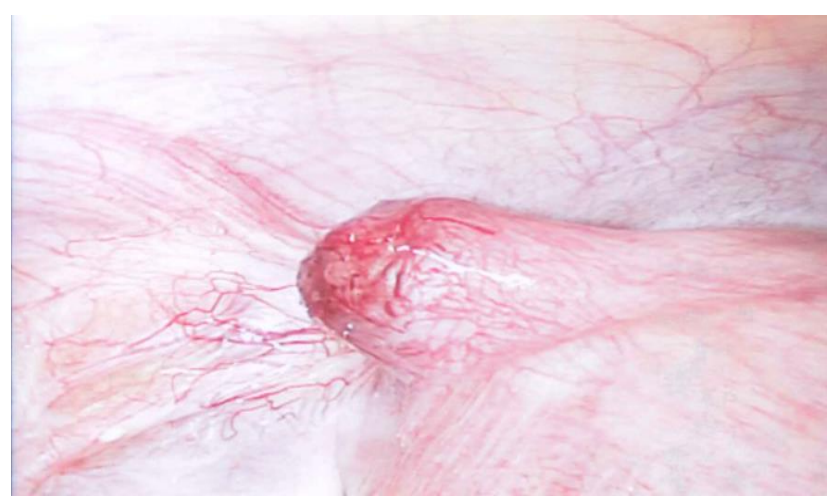

Figure 3: Left sided stump ectopic pregnancy.

\section{DISCUSSION}

The aetiology of stump ectopic pregnancy remains unclear. Several mechanisms have been proposed including the passage of spermatozoa through the patent fallopian tube and migration to the stump where fertilisation and implantation occurs. ${ }^{7}$ Takeda and colleagues suggest fertilisation of the ovum occurs in the patent tube with subsequent intrauterine migration into the stump, while another hypothesis is that the fallopian tube lamina remains intact at salpingectomy allowing later fertilisation and implantation within the stump. ${ }^{2,8}$ Based on this final hypothesis the same group recommend 'adequate' diathermy should be used at salpingectomy and that a hysterosalpingography (HSG) should be considered prior to the end of a salpingectomy to check tubal patency. If tubal patency is noted, they suggest occlusion devices such as coils can be considered. ${ }^{8}$ At this patient's previous salpingectomy bipolar was used to both remove the tube and diathermy the stump. A HSG was not performed. There appears to be no literature examining the risk of stump ectopic following use of energy devices versus simple ligation and incision.

There is no consensus on how much, if any stump remnant should be left at salpingectomy. Many gynaecologists believe that complete excision of the fallopian tube reduces recurrence. A literature review found no evidence for this. This case was reviewed with the surgeon who performed the initial salpingectomy. 
They stated their usual practice is to leave as little remnant stump as possible to reduce the risk of recurrence (Figure 4). A different surgeon who performed the recurrent ectopic operation suggested that if the remnant had not been so short the recurrence would have not been so close to the interstitial portion of the fallopian tube, and therefore the surgery would carry less risk. Another proposed reason for complete excision of the fallopian tube is to reduce future risk of tubal and ovarian cancer, however studies have shown these lesions most commonly occur in the distal portion of the tube. ${ }^{9}$ It should be noted that up to $20 \%$ of the ovarian blood supply is derived from the isthmic portion of the uterus. As such incision and/or diathermy in this region may affect ovarian reserve.

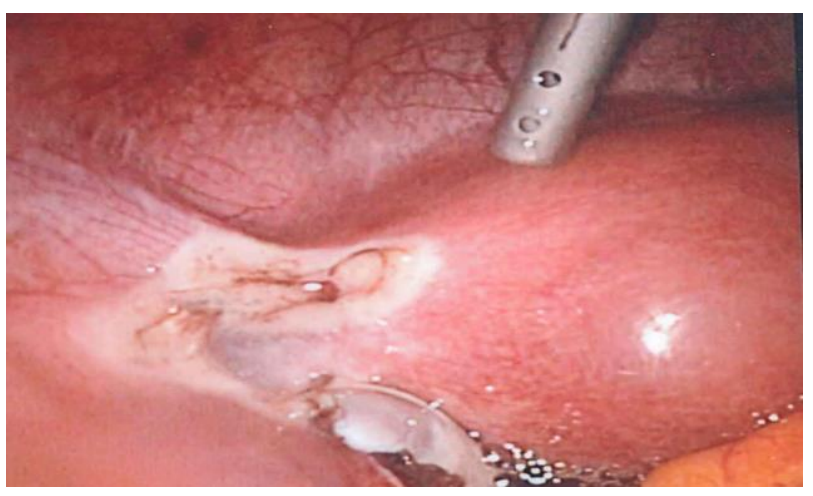

Figure 4: First salpingectomy of almost complete excision of tube.

Despite the site of this ectopic, blood loss was minimal. This may have been due to the use of argipressin. Argipressin is a synthetic peptide identical to endogenous antidiuretic hormone. It has marked vasoconstrictor properties. While vasopressors are widely used in medicine for their vasopressor action, other than case reports there appears to be only one trial demonstrating its safety and efficacy in salpingectomy. Ugur and colleagues compared argipressin to placebo in salpingectomy for ectopic pregnancy. ${ }^{10}$ They demonstrated a significant reduction in operative time and need for electro coagulation to secure haemostasis. There were no adverse effects documented. It is important to note that this trial was small and nonblinded, and argipressin has a significant number of side effects including anaphylaxis, bronchospasm and cardiac arrest.

\section{CONCLUSION}

Stump ectopic is a rare form of ectopic pregnancy and is potentially more dangerous than ampullary tubal ectopic pregnancy. There is no evidence currently regarding the best type of surgery for a first ectopic pregnancy with regards to the use of diathermy or length of remaining tube. It is our postulation that tubal remnants should be left long at salpingectomy for a first ectopic pregnancy. This is because we believe the recurrence rate is independent of tubal stump length and that recurrence in a long stump is preferable to a short stump as the bleeding risk with rupture is lower and the surgery is technically less difficult. However, further evidence in the form of randomised controlled studies of short vs long tubal remnants would be necessary to confirm or disprove this theory.

\section{Funding: No funding sources \\ Conflict of interest: None declared \\ Ethical approval: Not required}

\section{REFERENCES}

1. Anwar S, Uppal T. Recurrent viable ectopic pregnancy in the salpingectomy stump. Aus $\mathbf{J}$ Ultrasound Med. 2010;13(3):37-40.

2. Takeda A, Manabe S, Mitsui $T$, Nakamura $H$. Spontaneous ectopic pregnancy occurring in the isthmic portion of the remnant tube after ipsilateral adnexectomy: report of two cases. J Obstetr Gynaecol Res. 2006;32(2):190-4.

3. Ko PC, Liang CC, Lo TS, Huang HY. Six cases of tubal stump pregnancy: complication of assisted reproductive technology? Fertil Steril. 2011;95(7):2432-e1.

4. Souza C, Dullius T, Peters R, Genro V, Cunha-Filho JS. Importance of a making precocious diagnostic and implementing treatment of the ectopic pregnancy in the salpingectomy stump. Eur J Obstet Gynaecol Reproduct Biol. 2018;(223):141-2.

5. Zuzarte R, Khong CC. Recurrent ectopic pregnancy following ipsilateral partial salpingectomy. Singapore Med J. 2005;46(9):476.

6. Brincat M, Bryant-Smith A, Holland, TK. The diagnosis and management of interstitial ectopic pregnancies: a review. Gynecol Surg. 2019;(16)2.

7. Yano T, Ishida H, Kinoshita T. Spontaneous ectopic pregnancy occurring in the remnant tube after ipsilateral salpingectomy: a report of 2 cases. Reproduct Med Biol. 2009;8(4):177-9.

8. Samiei-Sarir B, Diehm C. Recurrent ectopic pregnancy in the tubal remnant after salpingectomy. Case Reports Obstet Gynaecol. 2013;2013.

9. George SH, Garcia R, Slomovitz BM. Ovarian Cancer: The fallopian tube as the site of origin and ppportunities for prevention. Front Oncol. 2016;6:108.

10. Ugur M, Yesilyurt H, Soysal S, Gokmen O. Prophylactic vasopressin duringlaparoscopic salpingotomy for ectopic pregnancy. J Am Asso Gynecol Laparoscop. 1996;3(3):365-8.

Cite this article as: Gaughran J, Mitchell S, Holland T. Salpingectomy for ectopic pregnancy: Does length really matter? Int J Reprod Contracept Obstet Gynecol 2021;10:333-5. 